\title{
Measuring effects on intima-media thickness: an evaluation of rosuvastatin in Chinese subjects with subclinical atherosclerosis-design, rationale, and methodology of the METEOR-China study
}

Yilong Wang ${ }^{1,2+}$, Anxin Wang ${ }^{1,2+}$, Hongwei $\mathrm{Li}^{3}$, Zhanquan $\mathrm{Li}^{4}$, Bo Hu${ }^{5}$, Xiaogang $\mathrm{Li}^{6}$, Huaguang Zheng ${ }^{1,2}$, Lu Fu', Hongtao Hu ${ }^{8}$, Zhiyu Nie ${ }^{9}$, Yulin Qin ${ }^{10}$, Bilian Zhao ${ }^{10}$, Di Wei ${ }^{10}$, Björn W. Karlson ${ }^{11,12}$, Michiel L. Bots ${ }^{13}$,

Yundai Chen ${ }^{14^{*}}$ and Yongjun Wang ${ }^{1,2^{*}}$

\begin{abstract}
Background: The beneficial effect of statins on atherosclerosis and cardiovascular outcomes has been well established. The Measuring Effects on intima media Thickness: an Evaluation Of Rosuvastatin (METEOR) global study demonstrated that a 2-year orally administered treatment with rosuvastatin $40 \mathrm{mg}$ daily significantly slowed the progression of carotid intima-media thickness (CIMT) compared to placebo. The current METEOR-China study is designed to evaluate the effect of rosuvastatin $20 \mathrm{mg}$ daily versus placebo on the progression of atherosclerosis measured by CIMT in asymptomatic Chinese subjects.

Methods: This is a phase 3, randomised, double-blind, placebo-controlled, multicentre parallel-group study. Asymptomatic Chinese subjects with a 10-year ischaemic cardiovascular disease (ICVD) risk $<10 \%$ will be recruited at 25 study sites. They will be treated with rosuvastatin $20 \mathrm{mg}$ or placebo for 104 weeks. The primary endpoint is the annualised rate of change in CIMT measured by B-mode ultrasonography. Secondary endpoints include the annualised rate of change in CIMT at three different sections of the carotid artery and changes in the serum lipid profile. Safety parameters will also be assessed.
\end{abstract}

Conclusion: The study will evaluate whether rosuvastatin $20 \mathrm{mg}$ slows the progression of CIMT in asymptomatic Chinese subjects at low risk of ICVD.

Trial registration: ClinicalTrials.gov NCT02546323. Registered on September 10, 2015

Keywords: Chinese, METEOR-China, Study design, Subclinical atherosclerosis, Rosuvastatin, Statin, Carotid intimamedia thickness

\footnotetext{
* Correspondence: cyundai@vip.163.com; yongjunwang1962@gmail.com

tWang Yilong and Wang Anxin contributed equally to this work.

${ }^{14}$ Department of Cardiology, China PLA General Hospital, 28 Fuxing Rd.,

Haidian District, Beijing 100853, China

${ }^{1}$ Beijing Tian Tan Hospital, Capital Medical University, Beijing, China

Full list of author information is available at the end of the article
}

(c) The Author(s). 2020 Open Access This article is licensed under a Creative Commons Attribution 4.0 International License, which permits use, sharing, adaptation, distribution and reproduction in any medium or format, as long as you give appropriate credit to the original author(s) and the source, provide a link to the Creative Commons licence, and indicate if changes were made. The images or other third party material in this article are included in the article's Creative Commons licence, unless indicated otherwise in a credit line to the material. If material is not included in the article's Creative Commons licence and your intended use is not permitted by statutory regulation or exceeds the permitted use, you will need to obtain permission directly from the copyright holder. To view a copy of this licence, visit http://creativecommons.org/licenses/by/4.0/ The Creative Commons Public Domain Dedication waiver (http://creativecommons.org/publicdomain/zero/1.0/) applies to the data made available in this article, unless otherwise stated in a credit line to the data. 


\section{Background}

During the last two decades, the mortality and morbidity caused by cardiovascular disease (CVD) have increased significantly in China [1]. Atherosclerosis is the most important cause of cardiovascular and cerebrovascular diseases. Furthermore, atherosclerosis may have a silent course for decades before symptoms and atherothrombotic complications occur [2], by which time the disease already has major histopathological consequences that are poorly reversible. Therefore, early treatment based on the risk factors and signs of subclinical atherosclerosis is necessary and important. According to a national cross-sectional study involving 107,095 residents aged $\geq 40$ years from the China National Stroke Prevention Project, for those 84,880 participants included in the analysis, the standardised prevalence of carotid atherosclerosis was $36.2 \%$, showing the potential clinical importance of focusing on primary prevention of atherosclerosis progression [3].

Low-density lipoprotein cholesterol (LDL-C) is a major risk factor for atherosclerosis $[4,5]$. Among lipid-lowering drugs, 3-hydroxy-3-methylglutaryl coenzyme A (HMG$\mathrm{CoA}$ ) reductase inhibitors (statins) are the cornerstone of therapy [6], and there is extensive evidence on the beneficial effects of statins on modification of the lipid profile, halting the progression of carotid and coronary atherosclerosis, and reduction of clinical cardiovascular morbidity and mortality [7-14]. Rosuvastatin is effective in lowering LDL-C and, like other statins, has favourable effects on other components of the lipid and lipoprotein profile, such as raising high-density lipoprotein cholesterol (HDL-C) and reducing levels of total cholesterol and triglycerides [15]. The principal evidence supporting the anti-atherosclerotic effects of rosuvastatin is based on the findings of the METEOR (Measuring Effects on intima media Thickness: an Evaluation Of Rosuvastatin; NCT00225589) study, the pivotal global phase 3 registration study that evaluated the effects of 2 years of treatment with rosuvastatin $40 \mathrm{mg}$ on the natural history of atherosclerosis of the carotid arteries in 984 patients from 61 centres in eight countries [16]. Patients enrolled in METEOR were at low risk (defined as 10-year Framingham Risk Index $<10 \%$ ) for coronary heart disease (CHD) according to the National Cholesterol Education Panel Adult Treatment Panel III (NCEP ATP III) 2001 guidelines and had subclinical atherosclerosis as assessed by carotid intima-media thickness (CIMT). Over the 2-year course of the study, rosuvastatin significantly slowed the progression of CIMT as compared with placebo. CIMT is considered a reliable surrogate marker for atherosclerosis [17], and using CIMT measurement as a marker for atherosclerosis may facilitate drug efficacy evaluation [18]. The major advantage of CIMT is that it is completely non-invasive and can be repeated as often as required.
Other small studies have shown the ability of rosuvastatin to slow the progression of atherosclerosis in Chinese and other Asian subjects. The REACH (Rosuvastatin Evaluation of Atherosclerotic Chinese Subjects) study showed that rosuvastatin may induce a rapid and lasting decrease in carotid plaque lipid content as assessed by magnetic resonance imaging [19]. In addition, the COSMOS (Coronary Atherosclerosis Study Measuring Effects of Rosuvastatin Using Intravascular Ultrasound in Japanese Subjects) study showed significant regression of coronary artery atheroma volume (assessed by intravascular ultrasound) in a Japanese patient population after a 76-week rosuvastatin treatment period [20].

The JUPITER (Justification for the Use of Statins in Prevention: an Intervention Trial Evaluating Rosuvastatin) outcome trial showed a significant $44 \%$ reduction in cardiovascular outcomes, including the reduction in mortality. JUPITER also showed that myopathy, hepatic injury, and cancer did not occur more frequently with rosuvastatin $20 \mathrm{mg}$ than with placebo during its median follow-up of approximately 2 years of 17,802 patients from 26 countries [21].

However, most of the evidence of the effects of rosuvastatin comes from studies conducted out of China. Race/ethnicity may impact the magnitude of the association between the risk factors and the presence of atherosclerotic disease [22]. Since Asian individuals are generally smaller in terms of height and body surface area than Caucasian individuals, the dosage of lipidmodifying drugs may be different in the two populations. Given these observations, the Chinese food and drug regulatory agency required evidence on the efficacy and safety obtained from studies in native Chinese populations.

We set out to conduct METEOR-China (ClinicalTrials.gov Identifier: NCT02546323) closely following the lessons learned from the global METEOR study. The principal objective of the METEOR-China study is to evaluate the effect of rosuvastatin $(20 \mathrm{mg} /$ day $)$ versus placebo on the progression of CIMT in asymptomatic Chinese subjects with subclinical atherosclerosis who have a 10-year ischaemic cardiovascular disease (ICVD) risk $<10 \%$. The design of this study is mainly based on that of the global METEOR study but includes only Chinese subjects, thereby complying with Chinese regulatory requirements by generating data from Chinese subjects (Schedule of activities [Fig. 1a] and SPIRIT checklist [supplementary Table 1] are included).

\section{Methods}

Study design

This is a phase 3, randomised, double-blind, placebocontrolled, multicentre, parallel-group study assessing the effects of rosuvastatin $20 \mathrm{mg}$ treatment for 104 weeks 
a

\begin{tabular}{|c|c|c|c|c|c|c|c|c|c|c|c|c|c|c|c|}
\hline \multirow[b]{3}{*}{ TIMEPOINTS } & \multirow{3}{*}{$\begin{array}{l}\text { Enrolment } \\
17 \text { Sep2015 } \\
\end{array}$} & \multirow{2}{*}{\multicolumn{3}{|c|}{$\begin{array}{l}\text { Allocation } \\
\text { (weeks) }\end{array}$}} & \multicolumn{10}{|c|}{ STUDY PERIOD } & \\
\hline & & & & & \multicolumn{9}{|c|}{ Post-allocation (weeks) } & \multicolumn{2}{|c|}{$\begin{array}{c}\text { Close-out } \\
\text { (weeks) }\end{array}$} \\
\hline & & -6 & -4 & -2 & 0 & 6 & 13 & 26 & 39 & 52 & 65 & 78 & 91 & 104 & 114 \\
\hline ENROLMENT: & $x$ & & & & & & & & & & & & & & \\
\hline Eligibility screen & & $x$ & $\mathrm{x}$ & $x$ & & & & & & & & & & & \\
\hline Informed consent & & $x$ & & & & & & & & & & & & & \\
\hline [List other procedures] & & & & & & & & & & & & & & & \\
\hline Allocation & & & & & $x$ & $\mathrm{x}$ & $x$ & $\mathrm{x}$ & $x$ & $x$ & $\mathrm{x}$ & $x$ & $\mathrm{x}$ & $\mathrm{x}$ & \\
\hline INTERVENTIONS: & & & & & 4 & & & & & & & & & $\rightarrow$ & \\
\hline [Rosuvastatin $20 \mathrm{mg}$ ] & & & & & $x$ & $x$ & $\mathrm{x}$ & $\mathrm{x}$ & $\mathrm{x}$ & $\mathrm{x}$ & $\mathrm{x}$ & $x$ & $\mathrm{x}$ & $x$ & \\
\hline [Placebo] & & & & & $x$ & $x$ & $x$ & $x$ & $x$ & $x$ & $x$ & $x$ & $x$ & $x$ & \\
\hline [List other study groups] & & & & & & & & & & & & & & & \\
\hline ASSESSMENTS: & & & $\leftarrow$ & & & & & & & & & & & $\rightarrow$ & \\
\hline [10-year ICVD risk < 10\%] & & & & & & & & & & & & & & & \\
\hline 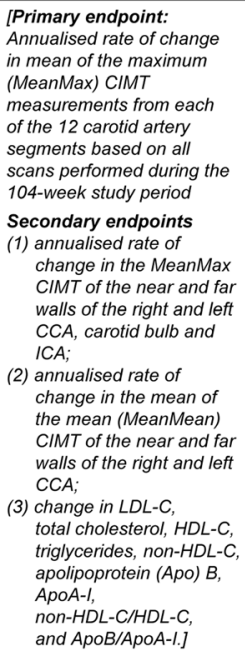 & & & $\mathrm{x}$ & $x$ & & & & $\mathrm{x}$ & & $\mathrm{x}$ & & $x$ & & $x$ & \\
\hline $\begin{array}{l}\text { [Safety assessments will } \\
\text { include adverse events } \\
\text { (AEs), serious adverse } \\
\text { events (SAEs), AEs } \\
\text { leading to discontinuation, } \\
\text { changes in clinical } \\
\text { laboratory analyses } \\
\text { (chemistry, haematology, } \\
\text { and urinalysis), vital signs, } \\
\text { 12-lead electrocardiograms } \\
\text { (ECGs), and physical } \\
\text { examination.] }\end{array}$ & & $x$ & $\mathrm{x}$ & $x$ & $x$ & $\mathrm{x}$ & $x$ & $\mathrm{x}$ & $x$ & $x$ & $\mathrm{x}$ & $x$ & $\mathrm{x}$ & $x$ & $x$ \\
\hline
\end{tabular}

b

Pre-randomised period

Randomised treatment period

$\begin{array}{lccccccccccccc}\text { Visit } & 1 & 2^{*} & 3^{*} & 4 & 5 & 6 & 7^{*} & 8 & 9^{*} & 10 & 11^{*} & 12 & 13^{*} \\ \text { Week } & -6 & -4 & -2 & 0 & 6 & 13 & 26 & 39 & 52 & 65 & 78 & 91 & 104\end{array}$

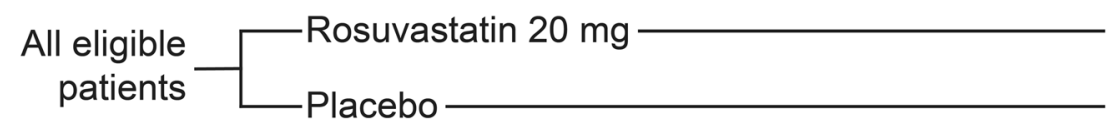

Fig. 1 a Schedule of activities. b Study flow chart. * Intima-media thickness measurement will be performed at visits 2, 3, 7, 9, 11, and 13. AE, adverse event; Apo, apolipoprotein; CCA, common carotid artery; CIMT, carotid intima-media thickness; ECG, electrocardiogram; HDL-C, highdensity lipoprotein cholesterol; ICA, internal carotid artery; ICVD, ischaemic cardiovascular disease; LDL-C, low-density lipoprotein cholesterol; MeanMax, mean of the maximum; MeanMean, mean of the mean; SAE, serious adverse event 
on the change in CIMT in adult Chinese subjects with subclinical atherosclerosis. It consists of 13 study visits: three screening visits, one baseline visit, and nine treatment visits (Fig. 1b). There will be 25 centres across China.

\section{Study population}

The study is designed to recruit asymptomatic Chinese subjects who have a 10 -year ICVD risk $<10 \%$. The ICVD risk is estimated based on the system of risk assessment introduced by the 2007 China Adult Dyslipidaemia Management Guidelines, in consideration of the Chinese ICVD prevalence characteristics that stroke is twice as prevalent as CHD [23]. According to this risk stratification system, the 10 -year ICVD risk is equivalent to $<10 \%$ when $\mathrm{LDL}-\mathrm{C}$ is $\geq 120 \mathrm{mg} / \mathrm{dL}(3.1 \mathrm{mmol} / \mathrm{L})$ and $<190 \mathrm{mg} / \mathrm{dL}(4.9 \mathrm{mmol} / \mathrm{L})$ for subjects without hypertension who have fewer than three other risk factors (including age), or when fasting LDL-C is $\geq 120 \mathrm{mg} / \mathrm{dL}$ $(3.1 \mathrm{mmol} / \mathrm{L})$ and $<160 \mathrm{~g} / \mathrm{dL}(4.1 \mathrm{mmol} / \mathrm{L})$ for subjects with only hypertension and age as ICVD risk factors, and subjects without hypertension who have three or more other risk factors (including age). Subjects need to have a maximum IMT of $\geq 1.2 \mathrm{~mm}$ and $<3.5 \mathrm{~mm}$ at any location in the carotid ultrasound scans. Detailed inclusion and exclusion criteria for the study are summarised in Table 1.

\section{Investigational products}

The investigational product will be rosuvastatin $20 \mathrm{mg}$ and placebo in oral tablet form for once daily use. The dose of $20 \mathrm{mg}$ rosuvastatin has been selected and agreed with the regulatory authority as it is the maximum approved rosuvastatin dose in China. Rosuvastatin exposure is approximately 2-fold higher in Asian populations than in Western populations at the same dose [24]. Thus, it is expected that the $20 \mathrm{mg}$ dose in Chinese people will yield similar exposure as $40 \mathrm{mg}$ in Westerners.

A placebo control has been chosen so that the normal change in IMT can be ascertained for subjects who meet the study entry criteria. These criteria were selected to provide a population of subjects whose low risk of cardiovascular events warrant therapeutic lifestyle changes according to the 2007 China Adult Dyslipidaemia Management Guidelines [23].

Rosuvastatin $20 \mathrm{mg}$ and the matching placebo will be prepared by the sponsor AstraZeneca and supplied in subject-specific labelled bottles. The randomisation schedule and treatment code will be assigned by an Interactive Voice Response System/Interactive Web Response System (IVRS/IWRS), and the site staff will dispense the investigational products to the study subjects accordingly.

\section{Modifications of the investigational products}

Subjects may be discontinued from the investigational product in the following situations: creatine kinase $>10 \times$ upper limit of normal (ULN) accompanied by muscle pain, tenderness, or weakness; alanine aminotransferase (ALT) or aspartate aminotransferase $(\mathrm{AST})>3 \times \mathrm{ULN}$; deterioration in the subject's condition that in the opinion of the investigator warrants subject withdrawal; and development of a condition that makes the subject highrisk, such as clinical atherosclerotic disease (myocardial infarction, transient ischaemic attack, stroke, angina pectoris, symptomatic coronary artery disease, peripheral arterial disease, abdominal aortic aneurysm) or diabetes mellitus. Subjects will be monitored to ensure that they are within the entry criteria limits for LDL-C throughout the study. If on two consecutive visits during the study LDL-C level exceeds the predefined range, the subject will be discontinued from the treatment.

\section{Concomitant medication}

Other medications considered necessary for the subject's safety and well-being may be given at the discretion of the investigator, provided that these medications do not conflict with the exclusion criteria. With the exception of lipid-regulating drugs, the only other disallowed drugs will be potent immunosuppressants, since the use of these agents may increase the risk of adverse events (AEs) during treatment with rosuvastatin.

\section{Efficacy endpoints}

CIMT assessment will be conducted at 12 carotid arterial segments: the near and far walls of the common carotid artery (CCA), the carotid bulb, and the internal carotid artery (ICA) of the right and left carotid arteries (Fig. 2).

The primary endpoint is the annualised rate of change in the mean of the maximum (MeanMax) CIMT measurements from each of the 12 carotid artery segments based on all scans performed during the 104-week study period. Secondary endpoints include the following: (1) annualised rate of change in the MeanMax CIMT of the near and far walls of the right and left CCA, carotid bulb, and ICA; (2) annualised rate of change in the mean of the mean (MeanMean) CIMT of the near and far walls of the right and left CCA; and (3) change in LDL$\mathrm{C}$, total cholesterol, HDL-C, triglycerides, non-HDL-C, apolipoprotein (Apo) B, ApoA-I, non-HDL-C/HDL-C, and ApoB/ApoA-I.

\section{Safety endpoints}

Safety assessments will include AEs, serious adverse events (SAEs), AEs leading to discontinuation, changes in clinical laboratory analyses (chemistry, haematology, and urinalysis), vital signs, 12-lead electrocardiograms 
Table 1 Detailed inclusion and exclusion criteria for METEOR-China

\section{Inclusion criteria}

- Provision of informed consent prior to any study-specific procedures

- Male aged $\geq 45$ and $<70$ years or female aged $\geq 55$ and $<70$ years

- Subjects with only hypertension (defined as blood pressure $\geq 140 / 90 \mathrm{mmHg}$ or on antihypertensive treatment) and age (male $\geq 45$ years, females $\geq 45$ years) as CVD risk factors and subjects without hypertension who have three or more other risk factors (including age) must have the following:

- Fasting LDL-C of $\geq 120 \mathrm{mg} / \mathrm{dL}(3.1 \mathrm{mmol} / \mathrm{L})$ and $<160 \mathrm{mg} / \mathrm{dL}(4.1 \mathrm{mmol} / \mathrm{L})$

- Subjects without hypertension who have fewer than three other risk factors (including age) must have the following: - Fasting LDL-C of $\geq 120 \mathrm{mg} / \mathrm{dL}(3.1 \mathrm{mmol} / \mathrm{L})$ and $<190 \mathrm{mg} / \mathrm{dL}(4.9 \mathrm{mmol} / \mathrm{L})$

- Triglycerides $<500 \mathrm{mg} / \mathrm{dL}(5.65 \mathrm{mmol} / \mathrm{L})$ at visit 1 (week - 6)

- HDL-C levels $\leq 60 \mathrm{mg} / \mathrm{dL}(1.6 \mathrm{mmol} / \mathrm{L})$ at visit 1 (week -6$)$

- Maximum IMT $\geq 1.2 \mathrm{~mm}$ and $<3.5 \mathrm{~mm}$ at any location in the carotid ultrasound scans conducted at both visit 2 (week - 4) and visit 3 (week - 2)

- Willing to follow all study procedures including study visits, fasting blood draws, and compliance with the study treatment regimen

\section{Exclusion criteria}

- Use of pharmacologic lipid-lowering medications (e.g. statins, fibrate derivatives, bile acid-binding resins, niacin, or its analogues at doses

$>400 \mathrm{mg}$ or prescribed Chinese traditional drugs), including CAls and CAl/statin combination, within 12 months prior to visit 1 (week -6)

- Current or recent (within 2 weeks of visit 1, week -6) use of supplements known to alter lipid metabolism (e.g. soluble fibres [including

$>2$ teaspoons Metamuci ${ }^{\circledR}$ or psyllium-containing supplement per day] or other dietary fibre supplements, marine oils, sterol/stanol products, or other supplement determined at the discretion of the investigator)

- History of hypersensitivity reactions to other HMG-CoA reductase inhibitors

- Pregnant women, women who are breastfeeding, and women of childbearing potential who are not using chemical or mechanical contraception or who have a positive serum pregnancy test

- Clinical evidence of CAD or any other atherosclerotic disease such as angina, MI, transient ischaemic attack, symptomatic CAD, cerebrovascular accident, percutaneous coronary intervention, coronary artery bypass graft, peripheral arterial disease, and abdominal aortic aneurysm

- History of cancer (other than basal cell carcinoma) in the past 2 years

- Uncontrolled hypertension defined as either a mean resting diastolic blood pressure of $\geq 110 \mathrm{mmHg}$ or a resting systolic blood pressure of $\geq 180$ $\mathrm{mmHg}$ recorded at any time during the screening period

- History of diabetes mellitus or current diabetes mellitus

- Uncontrolled hypothyroidism defined as a thyroid-stimulating hormone $>1.5$ times the ULN at visit 1 or subjects whose thyroid replacement therapy was initiated within the last 3 months

- History of heterozygous or homozygous familial hypercholesterolaemia or known hyperlipoproteinaemia types I, III, IV, or V (familial dysbetalipoproteinaemia)

- Use of the disallowed concomitant medications within 12 months prior to visit 1 (week - 6)

- History of alcohol and/or drug abuse within the past 5 years

- Active liver disease or hepatic dysfunction as defined by elevations of $\geq 1.5 \times$ ULN at visit 1 (week -6 ) in any of the following liver function tests: ALT, AST, or bilirubin

- Serum CK $>3 \times$ ULN at visit 1 (week - 6)

- Serum creatinine $>2.0 \mathrm{mg} / \mathrm{dL}(177 \mathrm{mmol} / \mathrm{L})$ recorded during the screening period

- Participation in another investigational drug study and having ingested investigational drug $\leq 4$ weeks before enrolment in the screening period

- Previous randomisation in the present study

- History of a significant medical or psychological condition that, in the opinion of the investigator, would compromise the subject's safety or successful participation in the study

- Involvement in the planning and/or conduct of the study (applies to both AstraZeneca staff and/or staff at the study site)

ALT alanine aminotransferase, AST aspartate aminotransferase, CAD coronary artery disease, CAl cholesterol absorption inhibitor, CK creatine kinase, CVD cardiovascular disease, HDL-C high-density lipoprotein cholesterol, HMG-COA 3-hydroxy-3-methylglutaryl coenzyme A, IMT intima-media thickness, LDL-C lowdensity lipoprotein cholesterol, $M I$ myocardial infarction, ULN upper limit of normal

(ECGs), and physical examination. All AEs, including SAEs, will be collected from the time of signature of the informed consent throughout the treatment period and within 10 days after the last dose of the study drug. ECGs will be taken at visits 4 and 13 . Laboratory safety assessments, vital signs, medical history, and physical examination will be performed to detect any sudden change in the condition according to the study plan shown in Table 2. 


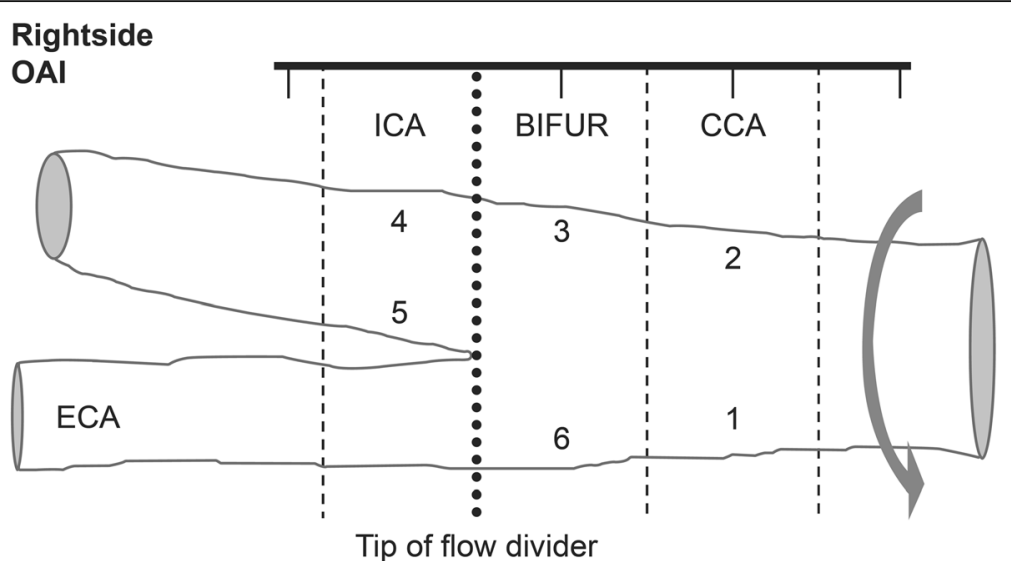

\section{Sequence of selections}

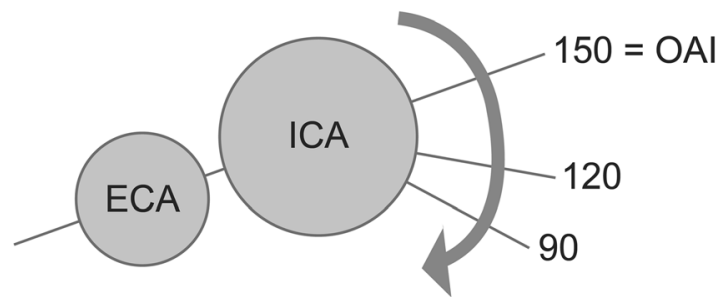

Fig. 2 Schematic of the left carotid artery in the longitudinal section. BIFUR, bifurcation; CCA, common carotid artery; ECA, external carotid artery; ICA, internal carotid artery; OAl, optimal anatomical interrogation angle

\section{CIMT measurements}

CIMT assessment will be conducted at visits $2,3,7,9$, 11, and 13. Carotid ultrasound images are recorded digitally by sonographers, and then sent to the Ward A. Riley Ultrasound Center, Wake Forest School of Medicine, Winston-Salem, USA, or Vascular Imaging Center of the UMC Utrecht, Utrecht, The Netherlands, for centralised evaluation. To ensure accuracy and consistency of the measurements between the study centres, all sonographers and readers will participate in a uniform training and certification programme before the start of the study. The design of the trial with duplicate baseline and duplicate end of study measurements provide for continuous availability of reproducibility information during the conduct of the trial for quality assurance and quality control purposes.

High-quality standardised longitudinal B-mode images will be obtained at 12 well-defined arterial wall segments in both the right and left carotid arteries. Each carotid artery has been divided into three segments (CCA, carotid bulb, and ICA) with six walls (near wall and far wall of each segment) as shown in Fig. 1. The three segments are defined as follows: the near wall and far wall of the arterial segment extending from 10 to $20 \mathrm{~mm}$ proximal to the tip of the flow divider into the CCA; the carotid bifurcation beginning at the tip of the flow divider and extending $10 \mathrm{~mm}$ proximal to the flow divider tip; and the $10 \mathrm{~mm}$ of the ICA distal to the tip of the flow divider.

The ultrasound examination will start with an initial exploratory transverse/longitudinal scan, followed by a detailed longitudinal examination of the specific arterial segments illustrated in Fig. 1. The exploratory scans are to help the sonographer become familiar with the participant's anatomy. During the transverse exploratory scan, the optimal anatomical interrogation (OAI) angle which best displays the tip of the flow divider and the "Y" appearance of the two arteries in a single longitudinal image can be determined. Based on the OAI angle with the tip of the flow divider positioned on the designated gridline, the sonographer can acquire a longitudinal exploratory image. Then, a detailed longitudinal scan for 12 segments at standardised angles can be performed. The images for each segment are selected at three predefined angles with $30^{\circ}$ increments on both sides: 90, 120, and 150 for the right side and 210, 240, and 270 for the left side. The images obtained will be stored as digital media or uploaded via the internet for offline measurement of CIMT.

The CIMT is determined as the distance from the interface between the vessel lumen and the intima to the interface between the media and the adventitia. The primary endpoint for the study is change in the average maximum CIMT over 12 carotid segments. 
Table 2 Efficacy and safety parameter assessment schedule

\begin{tabular}{|c|c|c|c|c|c|c|c|c|c|c|c|c|c|}
\hline \multirow{2}{*}{$\frac{\text { Study plan }}{\text { Visit number }}$} & \multicolumn{3}{|c|}{ Screening } & \multicolumn{10}{|c|}{ Treatment } \\
\hline & 1 & 2 & 3 & 4 & 5 & 6 & 7 & 8 & 9 & 10 & 11 & 12 & $\overline{13 / \mathrm{ET}^{\mathrm{a}}}$ \\
\hline Week number* & -6 & -4 & -2 & 0 & 6 & 13 & 26 & 39 & 52 & 65 & 78 & 91 & 104 \\
\hline Informed consent & $\checkmark$ & & & & & & & & & & & & \\
\hline Randomisation & & & & $\checkmark$ & & & & & & & & & \\
\hline Vital signs & $\checkmark$ & & & $\checkmark$ & $\checkmark$ & $\checkmark$ & & $\checkmark$ & & $\checkmark$ & & $\checkmark$ & $\checkmark$ \\
\hline Height & $\checkmark$ & & & & & & & & & & & & \\
\hline Body weight & $\checkmark$ & & & $\checkmark$ & & & & & & & & & $\checkmark$ \\
\hline Adverse events & & & & $\checkmark$ & $\checkmark$ & $\checkmark$ & $\checkmark$ & $\checkmark$ & $\checkmark$ & $\checkmark$ & $\checkmark$ & $\checkmark$ & $\checkmark$ \\
\hline Concomitant medications & $\checkmark$ & & & $\checkmark$ & $\checkmark$ & $\checkmark$ & $\checkmark$ & $\checkmark$ & $\checkmark$ & $\checkmark$ & $\checkmark$ & $\checkmark$ & $\checkmark$ \\
\hline Medical history & $\checkmark$ & & & & & & & & & & & & \\
\hline Physical examination & & & & $\checkmark$ & & & & & & & & & $\checkmark$ \\
\hline ECG & & & & $\checkmark$ & & & & & & & & & $\checkmark$ \\
\hline Chemistry panel & $\checkmark^{b}$ & & & $\checkmark$ & $\checkmark$ & $\checkmark$ & & $\mathfrak{v}^{c}$ & & $\checkmark^{c}$ & & $\mathfrak{\checkmark}^{c}$ & $\checkmark$ \\
\hline Pregnancy test ${ }^{d}$ & $\checkmark$ & & & & & & & & & & & & \\
\hline Haematology & & & & $\checkmark$ & & & & & & & & & $\checkmark$ \\
\hline Urine sample ${ }^{\mathrm{e}}$ & & & & $\checkmark$ & & & & & & & & & $\checkmark$ \\
\hline Serum lipid profile & $\checkmark$ & & & $\checkmark$ & $\checkmark$ & $\checkmark$ & & $\checkmark$ & & $\checkmark$ & & $\checkmark$ & $\checkmark$ \\
\hline$A p o A-l$ and $A p o B$ & & & & $\checkmark$ & & & & & & & & & $\checkmark$ \\
\hline Risk assessment & $\checkmark$ & & & & & & & & & & & & \\
\hline IMT & & $\checkmark^{f}$ & $\mathfrak{\checkmark}^{f}$ & & & & $\checkmark$ & & $\checkmark$ & & $\checkmark$ & & $\checkmark \mathfrak{V}^{9}$ \\
\hline Dispense investigational product & & & & $\checkmark$ & & $\checkmark$ & $\checkmark$ & $\checkmark$ & $\checkmark$ & $\checkmark$ & $\checkmark$ & $\checkmark$ & \\
\hline Investigational product compliance & & & & & $\checkmark$ & $\checkmark$ & $\checkmark$ & $\checkmark$ & $\checkmark$ & $\checkmark$ & $\checkmark$ & $\checkmark$ & $\checkmark$ \\
\hline TLC counselling ${ }^{h}$ & $\checkmark$ & & & $\checkmark$ & $\checkmark$ & $\checkmark$ & $\checkmark$ & $\checkmark$ & $\checkmark$ & $\checkmark$ & $\checkmark$ & $\checkmark$ & \\
\hline
\end{tabular}

$A L P$ alkaline phosphatase, $A L T$ alanine aminotransferase, Apo apolipoprotein, AST aspartate aminotransferase, CK creatine kinase, ECG electrocardiogram, ET early termination, IMT intima-media thickness, TLC therapeutic lifestyle changes, TSH thyroid-stimulating hormone

*The visit window for all study visits is \pm 7 days

${ }^{a}$ In the event of early termination, all non-IMT procedures scheduled for visit 13 (week 104) are to be conducted. A single IMT is to be performed on any subject who withdraws after 26 weeks

b Including TSH at visit 1

${ }^{\mathrm{C}}$ Abbreviated chemistry panel including liver function tests (ALT, AST, bilirubin, ALP), serum creatinine, and CK only

dUrine pregnancy test conducted at the study site. Required only for premenopausal women. Those with amenorrhoea for at least 1 year are exempt

${ }^{\mathrm{e}}$ For complete urinalysis

fIMT measurements at visit 2 (week - 4) and visit 3 (week - 2) must meet the inclusion criteria of maximum IMT $\geq 1.2 \mathrm{~mm}$ and $<3.5 \mathrm{~mm}$

${ }^{9}$ Final IMT procedures will be scheduled before discontinuation of study treatment. The second and final IMT procedures should occur at or before visit 13 (week

104), at the time of discontinuation of the study treatment. The two IMT procedures for visit 13 should be performed on different days when possible

${ }^{\mathrm{h}} \mathrm{TLC}$ counselling is to be reinforced at each clinic visit

We refer to this parameter as the MeanMax CIMT. For each segment, the maximum CIMT value is obtained by combining data across the three interrogation angles and selecting the largest CIMT measurement. Then, the estimate of MeanMax CIMT is computed by averaging the maximum CIMT values for the 12 carotid segments. For one of the secondary endpoints, the value of MeanMean CCA will be evaluated by averaging the mean CIMT values across the three angles of interrogation [16].

\section{Laboratory measurements}

Blood samples will be taken at the specified visits for analysis for serum lipids, haematology, pregnancy test, thyroid-stimulating hormone, and clinical chemistry (such as serum creatinine, liver function tests, creatine kinase). Subjects will have to fast for at least $8 \mathrm{~h}$ and will be seated for at least $5 \mathrm{~min}$ before blood samples are taken. Urine samples will be taken at specified visits.

\section{Statistical analysis \\ Sample size}

With 207 subjects per group, there will be $90 \%$ power to detect a difference of $-10.6 \mu \mathrm{m} /$ year in the change in the MeanMax CIMT of 12 vessel segments over a 104-week study period at a standard deviation of $33.28 \mu \mathrm{m} /$ year and a 0.05 two-sided significance level. Adjusting for an $18 \%$ dropout rate, based on the global METEOR study, a total of approximately 506 subjects are to be randomised. 
In the METEOR study, the difference in the annualised rate of change between the rosuvastatin and the placebo group was $-14.5 \mu \mathrm{m} /$ year [16]. The effect size was downregulated $10 \%$ to $-13.1 \mu \mathrm{m} /$ year, for a dose difference of $20 \mathrm{mg}$ rosuvastatin in the current study versus $40 \mathrm{mg}$ in the METEOR study. The 10\% downregulation in CIMT was selected based on the relative effect of 20 $\mathrm{mg}$ versus $40 \mathrm{mg}$ in LDL-C lowering in the VOYAGER meta-study [25]. The effect size was downregulated further $19 \%$ to $-10.6 \mu \mathrm{m} /$ year because of the ethnic difference of Chinese subjects in the current study versus Caucasian subjects in the METEOR study. The 19\% downregulation was selected based on the results from the MESA observational study [26] of CIMT over 10 years in different ethnicities in the USA.

\section{Randomisation and blinding}

Participants will be randomised by study investigators via the IVRS/IWRS to rosuvastatin $20 \mathrm{mg}$ or placebo. Randomisation will be stratified by ICVD risk $(<5 \%$ or 5 to $<10 \%)$. The randomisation scheme will be generated in blocks to ensure approximate balance (1:1) between the two treatment arms. The randomisation scheme will be produced by using the AstraZeneca global randomisation system, which incorporates a standard procedure for generating random numbers.

Subjects, investigators, study site personnel, sonographers, ultrasound image readers, and sponsor personnel involved with data review and analysis will remain blinded to the study treatment throughout the study. The study treatment will be blinded by providing rosuvastatin and matching placebo tablets that are indistinguishable from each other in appearance and that will be presented in the same packaging.

Medication will be labelled using a unique kit ID number, which is linked to the randomisation scheme. The investigators, via the IVRS/IWRS, will allocate randomisation numbers sequentially when sites call the IVRS/ IWRS to randomise an eligible subject. The IVRS/IWRS will also allocate the kit ID number to be dispensed to the subject.

AstraZeneca Patient Safety will have access in cases of emergency, and the drug supply chain will have access to enable supply of the investigational product. The central lab vendor will remain unblinded to the results of all laboratory tests. After screening, subjects and investigational site personnel will be blinded to blood lipid levels.

\section{Efficacy}

Three analysis sets will be used for data analysis: the intent-to-treat (ITT) population consists of all randomised subjects, the per-protocol (PP) population is a subset of the ITT population that includes subjects without any important protocol deviations, and the safety analysis set, which will consist of all subjects who take at least one dose of investigational product or placebo. The ITT population will be the primary efficacy analysis population. The CIMT analysis will be performed based on both the ITT and PP populations, and lipid and apolipoprotein analyses will be performed on the ITT population only. Safety parameters will be summarised on the safety analysis set.

A linear mixed effects (LME) model will be used for the primary analysis to assess the difference in the annualised rate of change in the CIMT measurements across 12 carotid artery sites between rosuvastatin $20 \mathrm{mg}$ daily and placebo treatment over 104 weeks. For the primary efficacy analysis, the dependent variable is the maximum CIMT measurement from three interrogation angles at each of the 12 carotid artery sites at each time point of CIMT measurement during the 104-week study period. The model includes fixed effects for treatment group, time, time-by-treatment interaction, ICVD risk stratification $(<5 \%$ or 5 to $<10 \%)$, age, sex, and scan reader, and random effects for the intercept and slope at the individual subject level and carotid artery site within subject. Ultrasound machine will be included as a fixed effect as well, if different types of machines are deployed across sites. For the time of CIMT measurement, the two pre-randomisation visits and the final two visits at the end of the study will be treated as separate time points. Details will be defined in the statistical analysis plan. Time, as a continuous variable, is the interval from the date of randomisation to the date of CIMT measurement. Differences in the annualised change between rosuvastatin and placebo will be evaluated by testing the time-by-treatment interaction term. In the analysis, let $\beta_{1}$ and $\beta_{2}$ represent respectively the coefficient parameters for time and time-by-treatment interaction in the mixed effect model. The annualised rate of change (slope) in MeanMax CIMT in placebo will be estimated by the coefficient parameter $\beta_{1}$, the annualised rate of change (slope) in MeanMax CIMT in rosuvastatin will be estimated by $\beta_{1}+\beta_{2}$, and the difference of the annualised rate (slope) of change between the two groups will be estimated by $\beta_{2}$. Then, the null and the alternative primary hypothesis can be expressed as $H 0: \beta_{2}=0$ versus Ha: $\beta_{2} \neq 0$ at a significance level of 0.05 two sided. No imputation of missing CIMT measurements will be performed based on previous work indicating that the LME modelling was the most optimal approach, with no added value of imputation of missing data [27].

The same statistical method used for the primary CIMT efficacy analysis will be applied to model the segment-specific secondary CIMT efficacy outcome measurements. The difference in the annualised rate of change between rosuvastatin and placebo for the secondary CIMT outcome measures will also be evaluated 
by testing the time-by-treatment interaction term in each model fitting. Secondary lipid and lipoproteins outcome measures are the percentage change from baseline, which will be analysed by analysis of covariance with terms for treatment in the model. For lipid measurements (LDL-C, total cholesterol, HDL-C, triglycerides, non-HDL-C, and non-HDL-C/HDL-C), both the analysis of the per cent change from baseline at the final visit and the analysis of the per cent change from baseline to time-weighted average during treatment will be performed. In the evaluation of change from baseline to the final visit, missing observations will be imputed by the last observation carried forward (LOCF). The timeweighted average lipid value is defined as the lipid value multiplied by the number of days since the last lipid assessment, summed for all lipid observations and divided by the sum of days between all visits. No imputation will be made for missing lipid values for time-weighted average analysis. For apolipoprotein measurements (ApoB, ApoA-I, and ApoB/ApoA-I), the analysis of the per cent change from baseline to final visit (LOCF) will be performed. No multiplicity adjustment will be applied to the secondary efficacy analysis.

\section{Safety}

Safety outcome measures including incidence of AEs (including SAEs and AEs leading to discontinuation), changes in clinical laboratory analyses (chemistry, haematology, and urinalysis), vital signs, 12-lead ECGs, and physical examination will be summarised descriptively. Safety analysis will be conducted using the safety analysis set. No formal statistical test will be performed.

\section{Data collection, management, and monitoring}

The Rave Web Based Data Capture (WBDC) system will be used for data collection and query handling. Investigators will ensure that data will be recorded on the electronic case report form as specified in the study protocol and in accordance with the instructions provided.

All sonographers will attend group quality control meetings to review study data and to maintain a high degree of standardisation of the data collection process throughout the study.

The data collected through third-party sources will be obtained and reconciled with study data. Data queries will be raised for inconsistent, impossible, or missing data. All entries to the study database will be available in an audit trail. The data will be validated as defined in a data management plan. Quality control procedures will be applied to each stage of data handling to ensure that all data are reliable and have been processed correctly.

During the study, an AstraZeneca representative will have regular contacts with the study site to ensure data integrity and the quality of trial conduct. Throughout the study, the quality control centres will continuously monitor the quality of the images submitted from the routine studies and the outcome variable data obtained from the within- and between-sonographer and withinand between-reader quality control studies.

\section{Ethics}

An Independent Ethics Committee (IEC) will approve the final study protocol, including the final version of the informed consent form (ICF), and any other written information and/or materials that are provided to the subjects. The investigator will ensure the distribution of these documents to the applicable IEC and to the study site staff.

This study will be performed in accordance with the ethical principles that have their origin in the Declaration of Helsinki and that are consistent with the International Council for Harmonisation (ICH)/Good Clinical Practice (GCP), applicable Chinese regulatory requirements, and the AstraZeneca policy on Bioethics and Human Biological Samples. The principal investigator(s) at each site will ensure each subject is given full and adequate oral and written information about the study and that they are provided a signed and dated informed consent before conducting any study-related procedure. A copy of the signed ICF will be given to each subject. Any protocol amendment will be communicated to the investigators, ethics committees, and other relevant parties.

\section{Discussion}

Several large, randomised, controlled studies have reported a beneficial effect of statin therapy on atherosclerosis [16, 28-33]. This effect of statins on carotid atherosclerosis was demonstrated using B-mode ultrasound measurement of CIMT, and on coronary atherosclerosis using quantitative coronary angiography and intravascular ultrasound. CIMT is commonly used [18], and has been recommended to assess overall CVD risk by the 2010 China Expert Consensus on Primary Prevention of CVD. The major advantage of CIMT is that it is completely non-invasive and can be repeated as often as required. It provides a continuous measure, since all subjects have a measurable carotid wall. It is also relatively inexpensive, and the technology is widely available [34]. Most important, CIMT is an accessible and reliable marker to assess subclinical atherosclerosis and cardiovascular risk [17]. For example, a meta-analysis of 119 randomised controlled trials involving 100,667 patients showed that the extent of interventions' effects on CIMT progression predicted the degree of CVD risk reduction [35]. Furthermore, a CIMT trial may considerably improve the efficiency of the evaluation of new drug therapies on atherosclerosis and CVD risk. Hence, the 
results of a CIMT trial can be seen as a decision tool to support the development of drugs targeting atherosclerosis [36].

The METEOR-China study is a prospective, randomised study designed to assess the effects of long-term treatment with rosuvastatin on CIMT in "low CHD risk" Chinese subjects with subclinical atherosclerosis. The low risk has allowed for a placebo comparison, and the placebo control ascertains the normal change in IMT for Chinese subjects who meet the entry criteria.

The dose of $20 \mathrm{mg}$ rosuvastatin has been selected for use in the current study as it is the maximum approved rosuvastatin dose in China (based on rosuvastatin exposure in Chinese patients). The intent is to arrest the progression of atherosclerosis to the same degree as was demonstrated in the METEOR trial, which used a dose of $40 \mathrm{mg}$ rosuvastatin [16]. Also, the METEOR-China study affords the opportunity to assess the long-term safety of the 20-mg dose with a placebo comparator in a Chinese population.

In conclusion, given the continuously rising prevalence and mortality of CVD in China, primary prevention of atherosclerosis progression is becoming more and more urgent. The METEOR-China study will ascertain whether rosuvastatin $20 \mathrm{mg}$ significantly slows the progression of subclinical atherosclerosis in addition to modifying serum lipid parameters, and assess the longterm safety profile of rosuvastatin $20 \mathrm{mg}$. The study has been designed to comply with the Chinese regulatory requirements by generating data from Chinese subjects, and thus bring new evidence and treatment options to reduce cardiovascular risk in Chinese people.

\section{Supplementary information}

Supplementary information accompanies this paper at https://doi.org/10. 1186/s13063-020-04741-0.

Additional file 1. SPIRIT Checklist: Recommended items to address in a clinical trial protocol and related documents.

\footnotetext{
Abbreviations

AE: Adverse event; ALT: Alanine aminotransferase; Apo: Apolipoprotein; AST: Aspartate aminotransferase; CCA: Common carotid artery; CHD: Coronary heart disease; CIMT: Carotid intima-media thickness; COSMOS: Coronary Atherosclerosis Study Measuring Effects of Rosuvastatin Using Intravascular Ultrasound in Japanese Subjects; CVD: Cardiovascular disease; ECG: Electrocardiogram; HDL-C: High-density lipoprotein cholesterol; HMG-CoA: 3-Hydroxy-3-methylglutaryl coenzyme A; ICA: Internal carotid artery; ICVD: Ischaemic cardiovascular disease; ITT: Intent-to-treat; IVRS: Interactive Voice Response System; IWRS: Interactive Web Response System; JUPITER: Justification for the Use of Statins in Prevention: an Intervention Trial Evaluating Rosuvastatin; LDL-C: Low-density lipoprotein cholesterol; LME: Linear mixed effects; LOCF: Last observation carried forward; METEOR: Measuring Effects on intima media Thickness: an Evaluation Of Rosuvastatin; NCEP ATP: National Cholesterol Education Pane Adult Treatment Panel; OAl: Optimal anatomical interrogation; PP: Perprotocol; REACH: Rosuvastatin Evaluation of Atherosclerotic Chinese Subjects; SAE: Serious adverse event
}

\section{Acknowledgements}

Editorial support was provided by Kerren Davenport, BSc, Prime, Knutsford, UK, in accordance with the Good Publication Practice guidelines (Link), and by Cactus Life Sciences (part of Cactus Communications), funded by AstraZeneca. Responsibility for opinions, conclusions, and data interpretation lies with the authors.

\section{Authors' contributions}

YJW and YDC conceptualised and designed the study. YLW and AXW drafted the initial manuscript, and all authors reviewed this manuscript critically. AXW, YLW, and YJW provided the data analysis plan. HWL, ZQL, BH, XGL, HGZ, LF, HTH, ZYN, YLQ, BLZ, DW, BWK, and MLB will be responsible for the recruitment, data collection, and data interpretation. The authors read and approved the final manuscript.

\section{Funding}

The METEOR-China study is funded by AstraZeneca. The AstraZeneca study team members were involved in designing the study and will be involved in the management of the study, interpretation of the data, and writing of the final report.

\section{Availability of data and materials}

Following AstraZeneca's transparency policy available at www. astrazenecaclinicaltrials.com

\section{Ethics approval and consent to participate}

The study protocol has been approved by the ethics committees at the study sites.

Written consent was obtained from every participant prior to any studyspecific procedures.

\section{Consent for publication}

This study will be published in scientific journals and be presented at conferences to target a wide range of audiences.

\section{Competing interests}

Yulin Qin, Bilian Zhao, Di Wei, and Björn W. Karlson are employees of AstraZeneca. Michiel L. Bots is indirectly supported by a grant from AstraZeneca for his contribution to the METEOR-China trial. Yilong Wang, Anxin Wang, Hongwei Li, Zhanquan Li, Bo Hu, Xiaogang Li, Huaguang Zheng, Lu Fu, Hongtao Hu, Zhiyu Nie, Yundai Chen, and Yongjun Wang have no competing interests to declare.

\section{Author details}

${ }^{1}$ Beijing Tian Tan Hospital, Capital Medical University, Beijing, China. ${ }^{2}$ China National Clinical Research Center for Neurological Diseases, Beijing Tiantan Hospital, Capital Medical University, No. 6 Tiantanxili, Dongcheng District, Beijing 100050, China. ${ }^{3}$ Department of Cardiology, Beijing Friendship Hospital Affiliated to Capital Medical University, Beijing, China. ${ }^{4}$ The People's Hospital of Liaoning Province, Shenyang, China. ${ }^{5}$ Department of Neurology, Union Hospital of Huazhong University of Science and Technology, Wuhan, China. ${ }^{6}$ Department of Neurology, Peking University Third Hospital, Beijing, China. ${ }^{7}$ The First Affiliated Hospital of Harbin Medical University, Harbin, China. ${ }^{8}$ Beijing Ji Shui Tan Hospital, Beijing, China. ${ }^{9}$ Department of Neurology, Shanghai Tongji Hospital, Tongji University School of Medicine, Shanghai, China. ${ }^{10}$ AstraZeneca R\&D, Shanghai, China. ${ }^{11}$ AstraZeneca R\&D Gothenburg, Mölndal, Sweden. ${ }^{12}$ Department of Molecular and Clinical Medicine, Institution of Medicine, Sahlgrenska Academy, University of Gothenburg, Gothenburg, Sweden. ${ }^{13}$ Julius Center for Health Sciences and Primary Care, University Medical Center Utrecht, Utrecht University, Utrecht, The Netherlands. ${ }^{14}$ Department of Cardiology, China PLA General Hospital, 28 Fuxing Rd., Haidian District, Beijing 100853, China.

Received: 12 March 2020 Accepted: 16 September 2020

Published online: 11 November 2020

\section{References}

1. Joint Committee for Developing Chinese Guidelines on Prevention and Treatment of Dyslipidemia in Adults. [Chinese guidelines on prevention and treatment of dyslipidemia in adults]. Zhonghua Xin Xue Guan Bing Za Zhi. 2007:35:390-419. 
2. Ross R. Atherosclerosis--an inflammatory disease. N Engl J Med. 1999;340: $115-26$.

3. Wang X, Li W, Song F, et al. Carotid atherosclerosis detected by ultrasonography: a national cross-sectional study. J Am Heart Assoc. 2018; 7(8):e008701.

4. Stamler J, Wentworth D, Neaton JD. Is relationship between serum cholesterol and risk of premature death from coronary heart disease continuous and graded? Findings in 356,222 primary screenees of the Multiple Risk Factor Intervention Trial (MRFIT). JAMA. 1986;256(20):2823-8.

5. Kannel WB, Castelli WP, Gordon T, McNamara PM. Serum cholesterol, lipoproteins, and the risk of coronary heart disease. The Framingham study. Ann Intern Med. 1971;74(1):1-12.

6. Grundy SM, Stone NJ, Bailey AL, et al. 2018 AHA/ACC/AACVPR/AAPA/ABC/ ACPM/ADA/AGS/APhA/ASPC/NLA/PCNA guideline on the management of blood cholesterol: a report of the American College of Cardiology/American Heart Association Task Force on Clinical Practice Guidelines. J Am Coll Cardiol. 2019;73(24):e285-350.

7. Scandinavian Simvastatin Survival Study Group. Randomised trial of cholesterol lowering in 4444 patients with coronary heart disease: the Scandinavian Simvastatin Survival Study (4S). Lancet. 1994;344:1383-9.

8. Shepherd J, Cobbe SM, Ford I, et al. Prevention of coronary heart disease with pravastatin in men with hypercholesterolemia. West of Scotland Coronary Prevention Study Group. N Engl J Med. 1995;333:1301-7.

9. Sacks FM, Pfeffer MA, Moye LA, et al. The effects of pravastatin on coronary events after myocardial infarction in patients with average cholesterol levels. N Engl J Med. 1996;335:1001-9.

10. Downs JR, Clearfield M, Weis $\mathrm{S}$, et al. Primary prevention of acute coronary events with lovastatin in men and women with average cholesterol levels: results of AFCAPS/TexCAPS. Air Force/Texas Coronary Atherosclerosis Prevention Study. JAMA. 1998;279:1615-22.

11. The Long-term Intervention with Pravastatin in Ischemic Disease (LIPID) Study Group. Prevention of cardiovascular events and death with pravastatin in patients with coronary heart disease and a broad range of initial cholesterol levels. N Engl J Med. 1998;339:1349-57.

12. Gotto AM Jr, Whitney E, Stein EA, et al. Relation between baseline and ontreatment lipid parameters and first acute major coronary events in the Air Force/Texas Coronary Atherosclerosis Prevention Study (AFCAPS/TexCAPS). Circulation. 2000;101:477-84.

13. Heart Protection Study Collaborative Group. The MRC/BHF Heart Protection Study of cholesterol lowering with simvastatin in 20,536 high-risk individuals: a randomized placebo-controlled trial. Lancet. 2002;56:53-6.

14. Sever PS, Dahlof B, Poulter NR, ASCOT investigators, et al. Prevention of coronary and stroke events with atorvastatin in hypertensive patients who have average or lower than-average cholesterol concentrations, in the Anglo-Scandinavian Cardiac Outcomes Trial-Lipid Lowering Arm (ASCOTLLA): a multicentre randomised controlled trial. Lancet. 2003;361:1149-58.

15. Karlson BW, Palmer MK, Nicholls SJ, et al. A VOYAGER meta-analysis of the impact of statin therapy on low-density lipoprotein cholesterol and triglyceride levels in patients with hypertriglyceridemia. Am J Cardiol. 2016; 117(9):1444-8.

16. Crouse JR 3rd, Raichlen JS, Riley WA, et al. Effect of rosuvastatin on progression of carotid intima-media thickness in low-risk individuals with subclinical atherosclerosis: the METEOR trial. JAMA. 2007;297:1344-53.

17. Onut R, Balanescu AP, Constantinescu D, et al. Imaging atherosclerosis by carotid intima-media thickness in vivo: how to, where and in whom? Maedica (Buchar). 2012;7:153-62.

18. Espeland MA, O'Leary DH, Terry JG, et al. Carotid intimal-media thickness as a surrogate for cardiovascular disease events in trials of HMG-CoA reductase inhibitors. Curr Control Trials Cardiovasc Med. 2005;6:3.

19. Du R, Cai J, Zhao XQ, et al. Early decrease in carotid plaque lipid content as assessed by magnetic resonance imaging during treatment of rosuvastatin. BMC Cardiovasc Disord. 2014;14:83.

20. Takayama T, Hiro T, Yamagishi M, et al. Effect of rosuvastatin on coronary atheroma in stable coronary artery disease: multicenter coronary atherosclerosis study measuring effects of rosuvastatin using intravascular ultrasound in Japanese subjects (COSMOS). Circ J. 2009;73:2110-7.

21. Ridker PM, Danielson E, Fonseca FA, et al. Rosuvastatin to prevent vascular events in men and women with elevated C-reactive protein. N Engl J Med. 2008;359:2195-207.

22. Giisberts CM, Groenewegen KA, Hoefer IE, et al. Race/ethnic differences in the associations of the Framingham risk factors with carotid IMT and cardiovascular events. PLoS One. 2015;10(7):e0132321. https://doi.org/10. 1371/journal.pone.0132321 eCollection 2015.

23. Chinese Society of Cardiology of Chinese Medical Association EBoCJoC. [Chinese guidelines for prevention of cardiovascular diseases]. Zhonghua Xin Xue Guan Bing Za Zhi. 2011;39:3-22.

24. Birmingham BK, Bujac SR, Elsby $R$, et al. Rosuvastatin pharmacokinetics and pharmacogenetics in Caucasian and Asian subjects residing in the United States. Eur J Clin Pharmacol. 2015;71:329-40.

25. Nicholls SJ, Brandrup-Wognsen G, Palmer M, Barter PJ. Meta-analysis of comparative efficacy of increasing dose of atorvastatin versus rosuvastatin versus simvastatin on lowering levels of atherogenic lipids (from VOYAGER). Am J Cardiol. 2010;105:69-76.

26. Tattersall MC, Gassett A, Korcarz CE, et al. Predictors of carotid thickness and plaque progression during a decade: the Multi-Ethnic Study of Atherosclerosis. Stroke. 2014;45:3257-62.

27. Peters $S A$, Bots $M L$, den Ruijter HM, et al. Multiple imputation of missing repeated outcome measurements did not add to linear mixed-effects models. J Clin Epidemiol. 2012;65(6):686-95.

28. Jukema JW, Bruschke AV, van Boven AJ, et al. Effects of lipid lowering by pravastatin on progression and regression of coronary artery disease in symptomatic men with normal to moderately elevated serum cholesterol levels. The Regression Growth Evaluation Statin Study (REGRESS). Circulation. 1995;91:2528-40.

29. Blankenhorn DH, Azen SP, Kramsch DM, et al. Coronary angiographic changes with lovastatin therapy. The Monitored Atherosclerosis Regression Study (MARS). Ann Intern Med. 1993;119:969-76.

30. Taylor AJ, Kent SM, Flaherty PJ, et al. ARBITER: Arterial Biology for the Investigation of the Treatment Effects of Reducing Cholesterol: a randomized trial comparing the effects of atorvastatin and pravastatin on carotid intima medial thickness. Circulation. 2002;106:2055-60.

31. Post Coronary Artery Bypass Graft Trial I. The effect of aggressive lowering of low-density lipoprotein cholesterol levels and low-dose anticoagulation on obstructive changes in saphenous-vein coronary-artery bypass grafts. N Engl J Med. 1997;336:153-62.

32. Nissen SE. Effect of intensive lipid lowering on progression of coronary atherosclerosis: evidence for an early benefit from the Reversal of Atherosclerosis with Aggressive Lipid Lowering (REVERSAL) trial. Am J Cardiol. 2005;96:61F-8F.

33. Corti R, Fuster V, Fayad ZA, et al. Effects of aggressive versus conventional lipid-lowering therapy by simvastatin on human atherosclerotic lesions: a prospective, randomized, double-blind trial with high-resolution magnetic resonance imaging. J Am Coll Cardiol. 2005;46:106-12.

34. Doneen AL, Bale BF. Carotid intima-media thickness testing as an asymptomatic cardiovascular disease identifier and method for making therapeutic decisions. Postgrad Med. 2013;125:108-23.

35. Willeit P, Tschiderer L, Allara E, et al. Carotid intima-media thickness progression as surrogate marker for cardiovascular risk: meta-analysis of 119 clinical trials involving 100,667 patients. Circulation. 2020;142:621-42. https:// doi.org/10.1161/CIRCULATIONAHA.120.046361.

36. Peters SA, den Ruijter HM, Grobbee DE, Bots ML. Results from a carotid intima-media thickness trial as a decision tool for launching a large-scale morbidity and mortality trial. Circ Cardiovasc Imaging. 2013;6:20-5.

\section{Publisher's Note}

Springer Nature remains neutral with regard to jurisdictional claims in published maps and institutional affiliations.

Ready to submit your research? Choose BMC and benefit from:

- fast, convenient online submission

- thorough peer review by experienced researchers in your field

- rapid publication on acceptance

- support for research data, including large and complex data types

- gold Open Access which fosters wider collaboration and increased citations

- maximum visibility for your research: over $100 \mathrm{M}$ website views per year

At $\mathrm{BMC}$, research is always in progress.

Learn more biomedcentral.com/submission 